\title{
Circadian rhythms in insect disease vectors
}

\author{
Antonio Carlos Alves Meireles-Filho ${ }^{1 /+}$, Charalambos Panayiotis Kyriacou² \\ ${ }^{1}$ Laboratory of Systems Biology and Genetics, Institute of Bioengineering, School of Life Sciences, \\ Ecole Polytechnique Fédérale de Lausanne, Lausanne, Switzerland 'Department of Genetics, University of Leicester, Leicester, UK
}

Organisms from bacteria to humans have evolved under predictable daily environmental cycles owing to the Earth's rotation. This strong selection pressure has generated endogenous circadian clocks that regulate many aspects of behaviour, physiology and metabolism, anticipating and synchronising internal time-keeping to changes in the cyclical environment. In haematophagous insect vectors the circadian clock coordinates feeding activity, which is important for the dynamics of pathogen transmission. We have recently witnessed a substantial advance in molecular studies of circadian clocks in insect vector species that has consolidated behavioural data collected over many years, which provided insights into the regulation of the clock in the wild. Next generation sequencing technologies will facilitate the study of vector genomes/transcriptomes both among and within species and illuminate some of the species-specific patterns of adaptive circadian phenotypes that are observed in the field and in the laboratory. In this review we will explore these recent findings and attempt to identify potential areas for further investigation.

Key words: circadian clock - disease vectors - gene expression - genomics

The Earth's rotation around its own axis as well as its angular tilt creates predictable cyclical changes in light and temperature with periods of $24 \mathrm{~h}$ and one year. These constant and predictable cycles have imposed a strong selective pressure on organisms to tune their internal physiology, metabolism and behaviour to these rhythmically fluctuating external conditions. Organisms have therefore evolved internal timekeepers to predict these daily and seasonal changes. The first report indicating that these rhythms were endogenously generated was published in 1729 by the French Astronomer Jean Jacques d'Ortous de Mairan. He observed that a sensitive heliotrope plant was able to show daily leaf movements even in the absence of environmental clues, i.e., in controlled laboratory conditions of constant darkness (de Mairan 1729). Because these rhythms are close to, but not exactly $24 \mathrm{~h}$, they are called circadian (Latin: circa $=$ about, dies $=$ day) and have been observed in organisms ranging from archaea to humans (Edgar et al. 2012), synchronising their physiology, metabolism and behaviour to the Earth's geophysical cycles.

Mosquitoes have contributed to chronobiology with many studies that characterise circadian phenotypes such as oviposition, sugar and blood feeding and locomotor activity (Clements 1999). As early as 1918, selfsustainable circadian flight activity rhythms in the mosquito Anopheles maculipennis were reported in controlled laboratory conditions (Roubaud 1918). In a study of spatial and temporal differences in the distribution of two Anopheline mosquitoes in Trinidad, West

doi: $10.1590 / 0074-0276130438$

+Corresponding author: acamf1@yahoo.com

Received 10 September 2013

Accepted 12 November 2013
Indies, Pittendrigh (1950) observed that although highly correlated with moisture conditions, the blood feeding activity persisted day after day in spite of overall humidity variation. The observation of this endogenous component in the wild, associated with evidence that circadian rhythms were genetically encoded (Bünning 1936), led Pittendrigh to study this phenomenon in detail using Drosophila as a model. After describing the circadian pattern of pupal-adult eclosion (Pittendrigh 1954), he artificially selected flies that eclosed either very early or very late in the daily distribution of emergence activity (Pittendrigh 1967). In the following years, numerous studies conducted by Pittendrigh and others helped to build up the conceptual model of the clock that gives rise to circadian rhythms [reviewed in Moore-Ede et al. (1982)]. Its main components are: (i) the "input or zeitgeber", which is any external stimulus that is transmitted to the internal pacemaker to adjust it with the environment, (ii) the pacemaker or biological clock, which is the endogenous oscillator that is self-sustainable, synchronisable and temperature compensated, and (iii) the "output", which is the behavioural and physiological periodic outcome from the interaction between the pacemaker and the input.

Main properties of the biological clocks - (i) Selfsustainability or free-running: even in the absence of periodical input from external factors, the endogenous rhythms persist with a period close to $24 \mathrm{~h}$; (ii) synchronisation: although independent from external factors, light, temperature and chemicals can "entrain" or adjust the clock period to match altered environmental conditions; (iii) temperature compensation: the freerunning period does not change over a wide range of temperatures, in contrast to most biochemical processes. Although this feature is conserved in all animals, it is especially relevant for poikilothermic animals such as insects, which change their body temperature over a day or among seasons. 
Over the last 30 years, many advances have been made towards understanding the molecular mechanisms of the circadian clock in the different kingdoms, including cyanobacteria (Synechococcus elongatus), filamentous fungi (Neurospora crassa), fruit flies (Drosophila melanogaster), plants (Arabidopsis thaliana), rodents and humans [reviewed in Dong et al. (2010), Baker et al. (2012), Nagel \& Kay (2012), Ozkaya \& Rosato (2012), Eckel-Mahan \& Sassone-Corsi (2013)]. On the other hand, although insect vectors were widely studied at the behavioural level in the last century, only recently have their clock molecules been elucidated. In the present review we will give a brief description of the Drosophila circadian clock as a comparative model to study the molecular clock of insect vector species and update several of the recent advances obtained in three insect vectors groups: triatomine bugs, phlebotomine sandflies and mosquitoes, highlighting their similarities and differences. We also discuss how the rich field of natural variation studies conducted in the past could be combined with recent next generation sequencing technologies to better understand these vector's clocks, which have an impact in the dynamics of diseases they transmit and therefore should be considered in future prevention and control programs (Klowden \& Zwiebel 2004).

The insect model: D. melanogaster - The isolation of the first period (per) clock mutants in D. melanogaster, heralded the first "behavioural" gene ever to be identified in any species by mutagenesis (Konopka \& Benzer 1971). As D. melanogaster is by far the best-understood "higher" organism at the genetic and molecular levels, with a bag of molecular/genetic tricks that cannot be equalled, much of what we know about the clock mechanism has understandably focused on this model species. The presence of Drosophila clock gene homologues in mammals indicates that the basic features of the circadian pacemaker are well conserved, although interactions among specific clock components vary (Gallego \& Virshup 2007). Insects thus share most of the fundamental clock features and the phylogenetic distance among them allows the use of D. melanogaster as a good starting point for dissecting the molecular clock in these species. In the following sections, we give a brief description of the D. melanogaster molecular clock, but as this is not our main focus we refer readers to a recent review that covers this topic in depth (Ozkaya \& Rosato 2012).

Clock anatomy - The circadian clock operates in a cell autonomous manner in almost all tissues in Drosophila (Plautz et al. 1997). While the central clock neurons located in the fly lateral brain regulate locomotor activity and mating rhythms (Helfrich-Förster 2004, 2005, Sakai et al. 2004, Helfrich-Förster et al. 2007), peripheral clocks found in the antennae, prothoracic glands (PGs), gut, Malpighian tubules etc. independently control their own circadian programs (Tomioka et al. 2012). Therefore, rather differently from mammals (and some insects) where the central clock in the brain synchronises the peripheral clocks, in Drosophila this control seems to be more relaxed. For example, electrophysiological responses to olfactory stimuli display a rhythm driven by clock genes expressed locally in the antenna, even when the fly's central clock is genetically ablated (Tanoue et al. 2004). Similarly, transplanted Malpighian tubules are able to keep self-sustained out-of-phase cycling for days within the host animal (Giebultowicz et al. 2000). These results suggest that multiple oscillators throughout the fly operate independently from a hierarchical system controlled by the brain via neural or humoral signals as observed in mammals. Nevertheless, internal temporal order is assured by direct light synchronisation (Plautz et al. 1997) assuring that their defined outputs act in unison to maintain physiological homeostasis.

The intracellular circadian pacemaker - Endogenous circadian rhythmicity is generated at the molecular level by interlocked transcriptional and translational feedback loops, which are autonomously operational in many cell types (Fig. 1). In the two main loops, the transcription factors coded by Clock $(\mathrm{Clk})$ and cycle ( $(\mathrm{yc})$ heterodimerise and bind to E-box (CACGTG) upstream regulatory regions of the genes per, timeless (tim), vrille (vri) and PAR-domain protein 1 ( $P d p 1)$, activating their expression (Fig. 2) (Hao et al. 1997, Allada et al. 1998, Darlington et al. 1998, Rutila et al. 1998, Cyran et al. 2003, Glossop et al. 2003). In the first loop, PER and TIM proteins start to accumulate in the cytoplasm at ZT12 [zeitgeber time where ZT0 is lights on and ZT12 is lights off in a light/dark (LD)12:12 cycle], about 6-8 $\mathrm{h}$ after their respective mRNAs. As PER alone is unstable, its accumulation is dependent of its heterodimerisation with TIM (Curtin et al. 1995, Gekakis et al. 1995, Zeng et al. 1996). Upon PER stabilisation by TIM, the PER/TIM heretodimer shuttles into the nucleus and indirectly shuts down tim and per expression by sequestering CLK/CYC from about ZT18-ZT4 (Lee et al. 1998, 1999, Bae et al. 2000, Yu et al. 2006). Without CLK/CYC mediated activation, per and tim levels start to decrease concomitantly with PER and TIM degradation, which consequently releases CLK/CYC inhibition, allowing a new round of transcription to occur. In a second loop, Clk transcription is regulated by VRI and PDP1, which compete for the same VRI/PDP1-boxes in the Clk promoter. While VRI represses $C l k$ transcription at around ZT14, PDP1 activates it around ZT18, which creates cyclical production of Clk (Cyran et al. 2003, Glossop et al. 2003) (Fig. 1). The pacemaker is also regulated at the posttranslational level by the kinases doubletime, shaggy, casein kinase 2 and the phosphatase PP2A, which have important roles in controlling the stability and nuclear entry of clock proteins (Price et al. 1998, Martinek et al. 2001, Lin et al. 2002, Sathyanarayanan et al. 2004). These transcriptional/translational regulatory loops generate circadian gene expression of its component molecules in different phases, except for $c y c$, which is constitutively expressed (Fig. 2). The periodic production of TFs is of vital importance for the clock output, as we discuss below.

Input - Light and temperature - Since endogenous rhythms are close to, but not equal to $24 \mathrm{~h}$, the pacemaker has to be adjusted on a daily basis by external factors such as light, temperature, chemicals and social cues to synchronise it to the environmental cycles (Ozkaya \& 
Rosato 2012). Among them, light was considered until recently to be the most important for entrainment and is the one best characterised at the molecular level.

The mechanism that light uses to reset the clock is through the blue light sensor Cryptocrome (CRY) (Emery et al. 1998, Stanewsky et al. 1998). Upon light activation, CRY binds to TIM and irreversibly commits it to degradation by the proteasome through the E3 ligase protein Jetlag (Ceriani et al. 1999, Naidoo et al. 1999, Busza et al. 2004, Koh et al. 2006, Peschel et al. 2006). In addition BRWD3, a substrate receptor for CRL4 (cullin 4 ring finger E3 ligase), has also been implicated in light induced CRY ubiquitinaytion and degradation (Ozturk et al. 2013). As PER is unstable without TIM, PER levels decrease and CLK/CYC inhibition is released, allowing a new cycle of transcription to take place (Hunter-Ensor et al. 1996, Myers et al. 1996, Zeng et al. 1996). Besides resetting the clock every morning, cells that autonomously express CRY can use this mechanism to phaseshift the clock to adapt to new environmental schedules (Ivanchenko et al. 2001, Rush et al. 2006) (Fig. 1).

Although light input was always recognised as the most important aspect for the clock entrainment, recent data suggests that in the wild temperature might be the most prominent zeitgeber (Vanin et al. 2012). Indeed, given its small size and poikilothermic nature, even small differences of $2-3^{\circ} \mathrm{C}$ are able to phase-shift the Drosophila rhythmic locomotor behaviour in constant darkness (Wheeler et al. 1993). So far, mutations in four genes have been isolated that impair temperature synchronisation: no-receptor-potential-A (norpA), nocte, transient receptor potential (TRP) cation channel $A 1$ and pyrexia $(P y x)$. Although their precise molecular mechanisms are not entirely clear, it is know that norp $A$ acts on the thermal-dependent regulation of per splicing, which increases PER levels that consequently advances the locomotor activity phase (Collins et al. 2004, Majercak et al. 2004). On the other hand, nocte appears to be important for proper structure and function of chordotonal organs (cho), which send temperature information to the brain, highlighting the importance of this sensory structure for temperature synchronisation (Sehadova et al. 2009). Pyx and TRP1 are TRP channels that function as thermo-receptors and, while $P y x$ is expressed in the cho, TPR1 is expressed in the brain (Lee \& Montell 2013, Wolfgang et al. 2013). Importantly, temperature reception in the brain is dependent on cho input (Sehadova et al. 2009), which highlights an interesting difference between light and temperature entrainment: while light can activate CRY in a cell autonomous manner, temperature acts on different receptors in different tissues and their coordination is important for the clock's thermal entrainment.

Output - Expression patterns - One of the ways by which the circadian pacemaker drives rhythmic output rhythms in behaviour, physiology and metabolism is by the circadian expression of downstream targets genes driven by the pacemaker transcription factors. Studies using DNA microarrays have conservatively estimated $10 \%$ of the Drosophila genome to be rhythmically expressed, covering a broad spectrum of cellular pathways (Claridge-Chang et al. 2001, McDonald \& Rosbash 2001, Ceriani et al. 2002, Lin et al. 2002, Ueda et al. 2002, Keegan et al. 2007). Among them, one study went further and compared the rhythmic expression in different

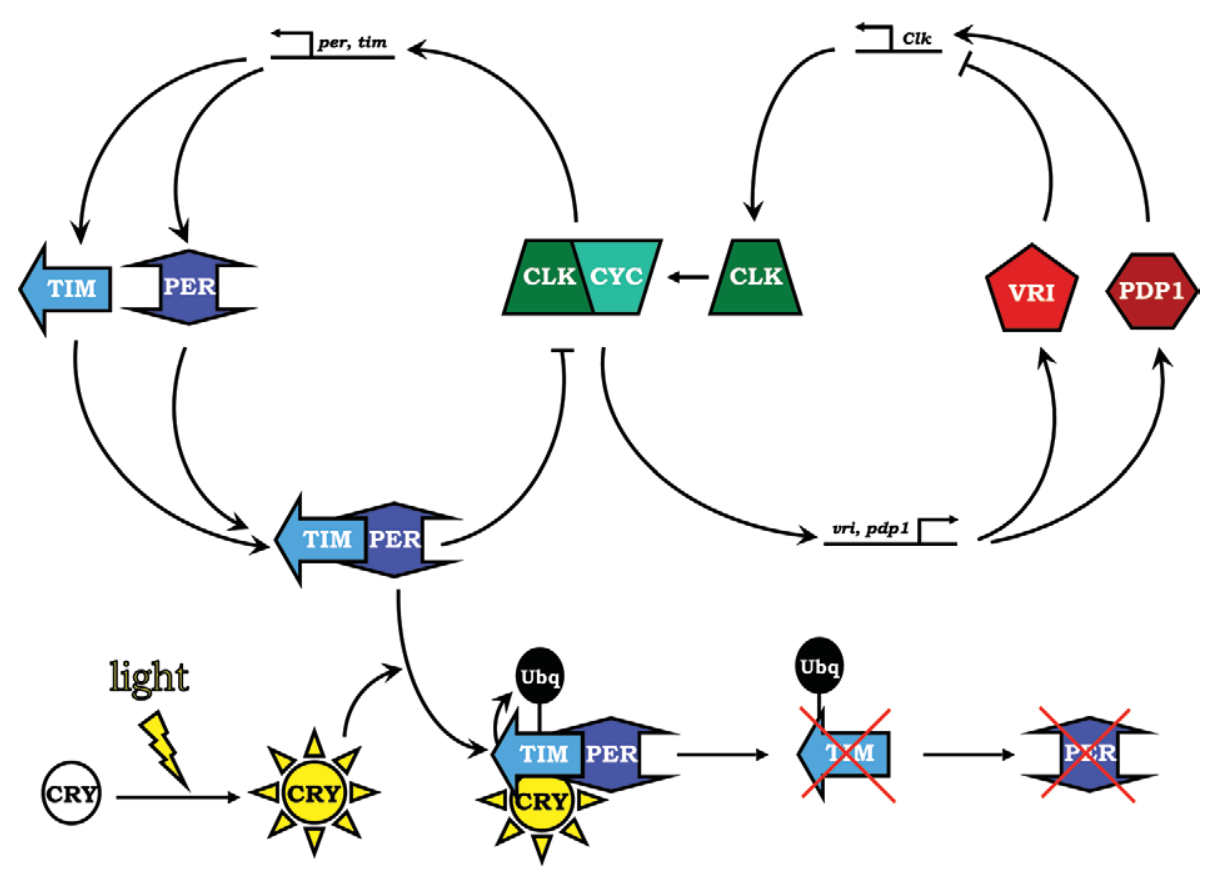

Fig. 1: model of the Drosophila melanogaster molecular clock comprising the two transcriptional/translational feedback loops mechanism and the light input pathway. CLK: Clock; CYK: Cycle; PDP1: PAR-domain protein 1; PER: Period; TIM: Timeless; Ubq: ubiquitin; VRI: Vrille. 
tissues, i.e. heads and bodies and identified that less than $10 \%$ of the cycling genes were shared between these datasets (Ceriani et al. 2002). This suggests that the clock control over output rhythms is highly tissue-specific and that genes that do not cycle in one tissue might cycle in another to drive tissue-specific physiological outputs. In agreement with that, more recent datasets on circadian expression in specific tissues have identified cycling genes correlated with tissues' specific functions. For example, most of the genes controlled by the circadian clock in the fly fat body are involved in physiological processes such as metabolism, steroid hormone regulation, detoxification and the immune response (Xu et al. 2011). In addition, the extent of the cycling transcriptome in clock cell neurons has revealed that the amplitude of cycling mRNAs in pigment dispersing factor expressing key pacemaker neurons is much higher than in other regions of the head (Kula-Eversole et al. 2010). Furthermore, in fly heads, more mRNAs cycle than nascent RNAs, suggesting a strong post-transcriptional component contributing to the cycling transcriptome (Rodriguez et al. 2013). These data highlight the importance of using more defined cell populations for a comprehensive "omics" understanding of the circadian clock.

Insect vector clocks - Insect vectors have revealed that a diverse number of events in their life cycle are controlled by an endogenous pacemaker. They have evolved particular circadian outputs that reflect adaptations for exploring specific environmental conditions as well as for coping with the resting periods of hosts and predators. Thus, the behavioural activity generally referred as "locomotor" in Drosophila comprises in insect vectors of a plethora of actions including sugar feeding, blood feeding, mating swarms and oviposition behaviours (Clements 1999, Saunders 2002, Barrozo et al. 2004b, Lazzari et al. 2004), all of which are important for de- termining the amount and quality of time spent with their hosts and thus critical for the dynamics of pathogen transmission. Recent years have witnessed a progression in moving from behavioural data (in the wild and controlled laboratory conditions) into physiology, genetics and molecular biology in these species. In the following sections, we will cover the advances in these fields for three groups of insect vectors: triatomine bugs, phlebotomine sandflies and mosquitoes.

Triatomine bugs - Triatomine bugs (Hemiptera: Reduviidae: Triatominae) are large bloodsucking insects that occur mainly in Latin America and the southern United States of America, where they are vectors of Chagas disease. Also known as American trypanosomiasis, this life-threatening zoonosis affects about seven-eight million people worldwide, most of them in Latin America where the disease is endemic. It is caused by the flagellated protozoan parasite Trypanosoma cruzi, which is transmitted by the faeces of infected triatomines through the insect sting.

Triatomine bugs are normally found in the burrows and nests of wild animals, on which they feed during the night by sucking blood. A number of species, among them the two major Chagas disease vectors Triatoma infestans and Rhodnius prolixus, have adapted to living in and near houses, where they feed on humans and domestic animals. They spend most of the daytime in an inactive state, or akinesis, hidden in their refuges. During the night they show two peaks of locomotor activity: one at dusk to search for the host and the second at dawn to take shelter (Lorenzo \& Lazzari 1998, 1999). Both peaks of activity are driven by the circadian clock and can be synchronised by light and temperature, but light is the predominant zeitgeber (Lazzari 1992). Interestingly, triatomines show an increased host's odour (carbon dioxide) perception around dusk, which persists in constant
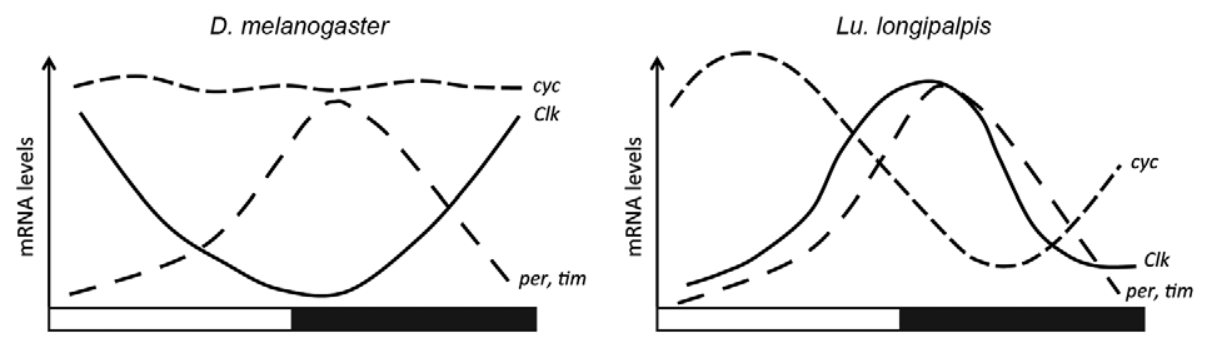

Ae. aegypti and Cx. quincefasciatus
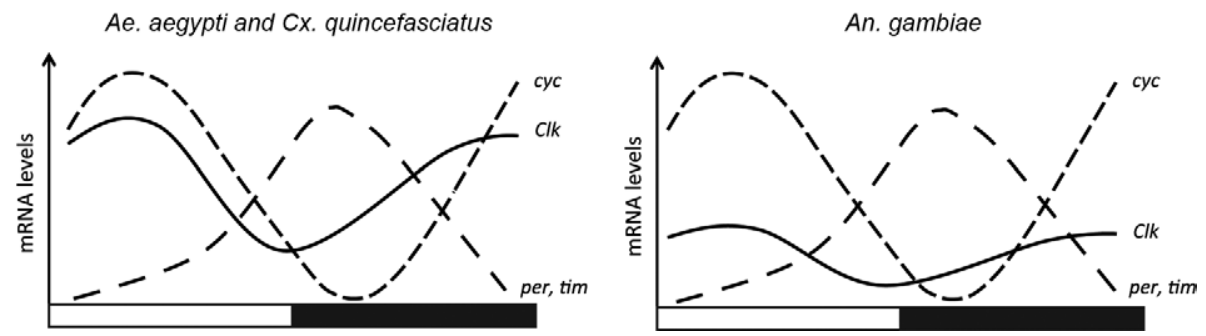

Fig. 2: circadian transcriptional profiles of the canonical clock genes in the main feedback loop from Drosophila melanogaster, Lutzomyia longipalpis, Aedes aegypti and Culex quinquefasciatus and Anopheles gambiae. Clk: clock; cyk: cycle; per: period; tim: timeless. 
conditions and is therefore also endogenously generated (Barrozo et al. 2004a, Bodin et al. 2008).

Besides locomotor activity, a number of other behaviours within the triatomines' life cycle are controlled by the circadian clock such as egg hatching, oviposition, ecdysis, sensitivity to light, thermopreference and light/ dark adaptation of compound eyes (Ampleford \& Steel 1982, Ampleford \& Davey 1989, Lazzari 1992, Reisenman et al. 1998, 2002, Minoli \& Lazzari 2003). One of the most dramatic changes in both morphological features and physiological regulatory mechanisms that are controlled by the clock in these species are moulting and metamorphosis (Steel \& Vafopoulou 2006), where in addition to their endocrine functions, prothoracicotropic hormone (PTTH) and ecdysteroids hormones are also key circadian outputs.

At the anatomical level, the circadian pacemaker in $R$. prolixus is composed of dorsal and lateral photosensitive clock neurons that rhythmically express PER and TIM and are probably analogous to D. melanogaster clock neurons that control rhythms in locomotor activity (Helfrich-Förster 2004, 2005, Helfrich-Förster et al. 2007). These neurons project close to axons of a group of neurosecretory cells that express the PTTH, providing timing information to the PTTH cells and other neurosecretory cells in the medial region of the protocerebrum (Vafopoulou \& Steel 1996, 2001, Vafopoulou et al. 2010). PTTH is a neuropeptide whose function is to stimulate the release of ecdysteroids by PGs (Warren et al. 1988). PG produces ecdysones, which are converted in peripheral tissues into 20-hydroxyecdysone (the major moulting hormone) whose titre leads to the initiation of the moulting process. The rhythmic release of PTTH not only stimulates, but also entrains the release of ecdysteroids by PG, which also possess light-entrainable autonomous oscillators (Steel \& Vafopoulou 2006). The resulting rhythm in haemolymph ecdysteroid titre is read by the target tissue's ecdysteroid nuclear receptors, which leads to internal tissue synchronisation and also triggers the rhythmic expression of downstream genes on different tissues such as fat body, rectal epithelium, oenocytes, neurosecretory cells and even the lateral clock neurons (Vafopoulou \& Steel 2006).

Besides controlling important aspects of triatomines development, this system also constitutes an interesting example of tissue temporal synchronisation that has striking differences compared to D. melanogaster and most insects analysed so far, where most peripheral oscillators are entrained directly by light (Giebultowicz 2000 ). In this sense, the $R$. prolixus circadian system resembles the mammalian one, where tissue-specific peripheral oscillators require input from the central clock in the brain to generate coherent systemic rhythms (Dibner et al. 2010), highlighting the potential importance of hormones as time signals in insects.

Phlebotomine sandflies - The sandfly Lutzomyia longipalpis (Diptera: Psychodidae: Phlebotominae) is the main vector of visceral leishmaniasis in the Americas (Deane \& Deane 1962). Leishmaniasis is caused by protozoan parasites from the genus Leishmania, which are believed to infect around 12 million people in 88 different countries. The transmission occurs when the infected females lacerate capillaries, inoculating pharmacologically active compounds to preserve the haemorrhagic pool upon which they feed, thereby realeasing the Leishmania parasites into the bloodstream.

In the wild sandflies are more active at dusk, which corresponds to the period when females seek a bloodmeal (Feliciangeli 2004). In fact, this pattern is also observed in controlled laboratory conditions and persists in constant darkness (Meireles-Filho et al. 2006b, Rivas et al. 2008). Blood-feeding, which is a known modulator of several mosquito species' behaviour (Clements 1999) also affects $\mathrm{Lu}$. longipalpis locomotor activity, reducing it at dusk in controlled laboratory conditions. This apparently involves the central pacemaker, since per and tim expression levels were not only reduced in the female body, but also in the head (Meireles-Filho et al. 2006b).

Lu. longipalpis was the first insect vector to have a clock gene identified (per) (Peixoto et al. 2001) and the subsequent cloning of other clock genes and expression analyses of molecular members from the first feedback loop showed considerable similarities, but also interesting differences compared to D. melanogaster. While per and tim mRNAs cycle, peaking around ZT13 in sandflies, as in flies and other insects (So \& Rosbash 1997, Goto \& Denlinger 2002, Meireles-Filho et al. 2006b, Sandrelli et al. 2008), Clk expression peaks around ZT 9-13, about half a day later than in D. melanogaster (Bae et al. 1998, Lee et al. 1998, Meireles-Filho et al. 2006b). In addition, $c y c$ expression cycled robustly, beginning to rise at the end of the night and peaking in the middle of the day ZT 5-9 (Meireles-Filho et al. 2006a), in sharp contrast with Drosophila where cyc is constitutively expressed (Bae et al. 2000) (Fig. 2). Another notable difference observed in the sandfly clock is the C-terminal transactivation domain found encoded in the $c y c$ gene that is absent in Drosophila (Rutila et al. 1998, MeirelesFilho et al. 2006a). It is believed that during the course of evolution Drosophila has compensated for the lack of this domain by acquiring an extensive poly-Q transactivation domain in the Clk gene, thereby keeping CLK/ CYC heterodimer functionality (Allada et al. 1998).

But although several transcriptome studies have identified many new sandfly molecules (Anderson et al. 2006, Dillon et al. 2006, Jochim et al. 2008, Oliveira et al. 2009, Pitaluga et al. 2009, Azevedo et al. 2012), the lack of the full sequence genome of this vector has hindered further advances in the molecular details of its circadian clock. As the current Lu. longipalpis genome project has already sequenced about 22.6 million reads (representing a coverage of 38.9x) (vectorbase.org), we expect that these efforts will help to boost our knowledge in the sandfly circadian clock in the future.

Mosquitoes - Mosquitoes (Diptera: Culicidae) are remarkably adaptive species and some species are important vectors of harmful human diseases such as malaria, yellow fever and filariasis. Their circadian rhythms have been extensively studied, both in the wild and in laboratory conditions. They show a remarkable variation of 
locomotor activity behaviours that can be affected by several factors such as nutritional and mating status, temperature and light intensity (Clements 1999). In addition, there is increasing evidence that mosquito behaviour can also be modulated by parasites to increase transmission rates (Hurd 2003, Schaub 2006). This makes the study of these insects' circadian rhythms of epidemiological relevance and important for disease control. In addition, the availability of fully sequenced genomes from phylogenetically closely related species with different circadian rhythms makes mosquitoes valuable for the molecular analysis of these processes. Given the large amount of data that could be potentially cited, we have decided to focus the following section on the species that have their genomes fully sequenced and whose circadian clocks are best characterised at the molecular level: Culex quinquefasciatus, Aedes aegypti and Anopheles gambiae.

C. quinquefasciatus - Mosquitoes from the Culex genus are the most diverse and geographically widespread of these three mosquito genera. They are the main vectors of West Nile encephalitis, St. Louis encephalitis and most importantly nematodes that cause lymphatic filariasis (LF). LF is currently endemic in 72 countries and is caused by three species of nematode: Wuchereria bancrofti, Brugia malayi and Brugia timori, which are transmitted to the hosts through the bite of infected female mosquitoes. Interestingly, the interaction between Culex and the filarial worm is one of the best examples of coevolution of circadian rhythms of parasitism and its adaptive value: the worms reach their highest density in the human peripheral blood at the time when the vector shows its biting activity peak, increasing their chances of transmission into the insect (Hawking 1975). Culex presents a nocturnal pattern of activity in the wild that can be reproduced under controlled laboratory conditions (Jones 1982, Gentile et al. 2009) and can be synchronised by temperature cycles (Chiba et al. 1993). In addition it is and modulated by insemination status (Chiba et al. 1990), which is driven by a pheromone produced by the male accessory gland (Jones \& Gubbins 1979).

The molecular analysis of the biological clock in this species assessed the structure and circadian expression of genes of the two main loops. Besides the presence of per, tim, Clk, cyc, vri and Pdpl, two cry genes were cloned in Culex. This was also observed in other species and this is apparently the most ancestral organisation of circadian systems in insects where CRY1 serves as a photic synchroniser as in Drosophila and CRY2 participates in the core clock as a repressor of CLK/CYCmediated gene transcription as in mammals (Zhu et al. 2005, 2008, Yuan et al. 2007). In general, the circadian expression patterns were similar with those observed in other insects, with some exceptions. For example in the first loop, while $C l k$ expression peaked around ZT22ZT2, similar to Clk expression in Drosophila and opposite to $\mathrm{Lu}$. longipalpis, cyc expression was rhythmic as in sandflies and peaked slightly earlier at ZT1-5 (Fig. 2). Interestingly, the expression of the cry genes differed: while the photoreceptor cryl was constitutive (in contrast to Drosophila cry that peaks at ZT5) (Zheng et al. 2008), cry2 cycled with a peak at ZT15-19 (Gentile et al. 2009). The molecular structure of the Culex clock with its two cry genes suggests that cry2 will represent a negative regulator, but whether the two cry's could be involved in the differences observed in the locomotor activity rhythms among species remains open (Gentile et al. 2009) (see below).

Ae. aegypti is the best-characterised species within the Culicinae and is the primary vector for yellow fever and dengue. Dengue fever is caused by the dengue virus, which is transmitted to humans through the bites of infected female mosquitoes. The disease occurs in tropical and sub-tropical regions and has recently advanced into urban and semi-urban areas. It is estimated that 50-100 million dengue infections occur worldwide every year, which makes this disease a major international public health problem.

Ae. aegypti - Ae. aegypti is considered an urban mosquito and shows endophilic and anthropophilic behaviour, being frequently caught inside houses and feeding on human blood. It has diurnal rhythms of blood-feeding activity that are modulated by reproductive state (i.e., insemination) and feeding status. Virgin females show a bimodal pattern of activity, which decreases after they mate. When mated and blood-fed, they become inactive for the first two days after the blood-meal, but recover activity on subsequent days, probably reflecting the search for a place to oviposit (Taylor \& Jones 1969, Jones 1981).

As with Culex, orthologues of Drosophila circadian genes were also cloned in Ae. aegypti and had their daily expression analysed (Gentile et al. 2006, 2009). Analysis of circadian abundance of the genes from the two loops revealed striking similarities with that observed for Culex, despite the fact that they have different locomotor activity patterns (Aedes diurnal vs. Culex nocturnal) (Fig. 2). Aedes also has two cry genes and the expression of $c r y 2$ was the main difference between these two species: while Culex had a peak of expression at ZT15-19, Aedes cry2 had a bimodal peak at ZT1-5 and ZT15-19, raising the interesting possibility that $c r y 2$, which is homologous to the mammalian-like repressor cry, might be responsible for the differences observed in locomotor activity behaviour between these two species and among other insect species in general (Gentile et al. 2009).

The publication of the Ae. aegypti genome provoked a number of studies that have addressed the transcriptional profile of this species in development, insecticide challenge and after blood-feeding [reviewed in Severson \& Behura (2012)]. Ptitsyn et al. (2011) analysed genomewide rhythmic expression in female heads using Agilent microarrays under LD conditions and have found that molecules in a number of important pathways can cycle. For example, genes related to detoxification pathways such as xenobiotic metabolism and cytochrome P450 family were expressed with a period of $24 \mathrm{~h}$ (Ptitsyn et al. 2011), which might explain the daily fluctuation of permethrin-resistance (Yang et al. 2010) and therefore be considered in insecticide control programs. As this study did not assess cyclic expression in constant conditions [dark/dark (DD)], it is likely that many of the observed rhythmically expressed gene are a direct response to light instead of being controlled by the circa- 
dian clock. Indeed, $59 \%$ of rhythmic genes in a similar study with An. gambiae were rhythmic only under LD conditions (Rund et al. 2011) (see below).

An. gambiae - The mosquito An. gambiae is the primary African malaria vector, which is caused by parasites from the Plasmodium genus. As for LF and dengue, the parasites are transmitted through the bites of infected Anopheles female mosquitoes and about 219 million cases of malaria were estimated in 2010 with approximately 660,000 deaths.

The mosquito daily behavioural rhythms include dusk mating swarms, nocturnal flight activity, sugar feeding, blood feeding and oviposition. It is a nocturnal species and, as for Culex and Aedes, its rhythms are also modulated by insemination and blood-feeding (Jones et al. 1972, Jones \& Gubbins 1978, Gary \& Foster 2006, Das \& Dimopoulos 2008).

Analysis of clock gene circadian expression in $A n$. gambiae revealed a pattern indistinguishable from that observed with Culex (Fig. 2), including constitutive cryl expression and unimodal cry2 rhythmic expression (Rund et al. 2011). Since An. gambiae is a nocturnal species as is Culex, it is tempting to speculate that cry2 expression indeed might be responsible for the differences observed in locomotor patterns among insect species. However, this can only be definitively answered with the type of interspecific transformation experiments that have been carried out in Drosophila to dissect the molecular basis of species-specific locomotor profiles (Tauber et al. 2003).

To better understand the molecular interactions among the circadian clock, light sensing system and host seeking, Das and Dimopoulos (2008) measured genome wide expression changes after the application of light pulses at the beginning of night, which is known to inhibit blood-feeding behaviour. They observed that while short light pulses inhibit blood-feeding in a clock independent manner, long light stimulation induced phase advances in blood-feeding propensity in a clock dependent manner. Interestingly, both light regimes altered the expression of clock related genes such as tim and the takeout gene family (which links circadian locomotor rhythms and feeding behaviour in Drosophila) (SarovBlat et al. 2000, So et al. 2000). Likewise, silencing of tim, cry (which like tim is also involved in the clock's synchronisation by light) and takeout genes promoted an increase propensity for the mosquito to feed on blood, suggesting that they are important components of the circadian clock that drives blood-feeding activity (Das \& Dimopoulos 2008). Besides these genes, the light treatment induced transcriptome changes in a variety of physiological systems similar to the ones caused by blood-feeding, highlighting the possible role of the circadian clock in linking these two processes.

Several other studies have addressed the global circadian regulation of gene expression in this species also in $\mathrm{DD}$, where rhythmic gene expression in diverse biological functions such as metabolism, detoxification, olfaction, vision, cuticle/peritrophic membrane regulation and immunity have been uncovered, all of them tissue-specific (Rund et al. 2011, 2013). The comparison between the
An. gambiae and Ae. aegypti circadian transcriptomes revealed that genes related to visual signalling pathways cycled with somewhat similar phases, suggesting that sensitivity tuning to photic activation does not depend on the temporal niche (i.e. nocturnal vs. diurnal). In contrast, odorant binding protein (OBP) genes, important components of the olfactory system, differed in that $A n$. gambiae OBPs expression showed a very tight regulation with all 17 genes clustered around ZT9-13, while in Ae. aegypti the 15 mRNAs identified peaked in expression at various times of the day (Rund et al. 2013).

Are species-specific differences in behaviour and physiology directly controlled by the core pacemaker or reflect differences in how the information of the central clock is translated into output signals? In light of these datasets, it is tempting to speculate that changes in the transcriptional downstream programs are more likely due to the differences in the regulatory sequences of upstream clock target genes than changes in the main clock components as seen in other systems (Meireles-Filho \& Stark 2009). On the other hand, cry2 differential expression might be an important factor in this equation and was also found to affect output rhythms in diverse species. For example, in two strains of Bactrocera cucurbitae that differed in mating time, the allele frequency at two polymorphic sites in cry, as well as cry gene expression itself, correlated with differences in circadian locomotor period, suggesting that variation in the cry gene is related to differences in the circadian behaviour in the two strains (Fuchikawa et al. 2010). Even in plants a single amino-acid substitution from methionine to valine in the cry2 gene was associated with an early flowering quantitative trait loci (El-Din El-Assal et al. 2001). Future studies on the biochemical and molecular interactions between clock genes as well as interspecific transformation experiments in loss-of-function $D$. melanogaster mutants [e.g. Tauber et al. (2003)], might further illuminate the role of clock genes on activity and their modulation by mating and feeding status.

Future directions - Although the circadian clock of insect vectors control a number of aspects of their behaviour and physiology important for their survival, including sensory perception and susceptibilities to insecticide or immune challenge, the temporal organisation of the vectors' life has not yet been exploited by vectorcontrol programs. This is in part hindered by the fact that it is still unclear how the circadian clock connects to output rhythms, and therefore any strategy considering manipulation of physiology and behaviour through the clock would probably not succeed.

The advancement in next generation sequencing technologies provides means by which association of a phenotype to its causal polymorphism is now relatively straightforward [e.g. Massouras et al. (2012)]. Allied to the extensive natural circadian activity variation of insect vectors found in the wild that can be reproducibly observed in the laboratory [e.g. Rivas et al. (2008), Rund et al. (2012)], this scenario configures an exciting opportunity to link genetic and phenotypic variation, which might lead us to better understand the molecular 
underpinnings of the circadian clock, its variation and how plasticity is achieved. As the clock is fundamentally important to every aspect of behaviour and physiology of almost all higher organisms, the circadian analysis of insect vectors, given their epidemiological importance, must not be undervalued by the vector community.

\section{ACKNOWLEDGEMENTS}

This article is written and dedicated to the memory of Alexandre Afranio Peixoto (1963-2013), student of CPK and mentor of ACAMF. Alex lit up the population genetic and molecular evolution study of insect clock genes in the 1990's with a series of outstanding papers, mostly on the period gene of Drosophila. He used the newly developed neutrality tests of that time to study the evolutionary signatures present in the patterns of molecular variation in and around clock genes and was able to determine whether natural selection was shaping that variation. However unlike almost all others, he allied this statistical approach to DNA sequences with phenotypic analyses and generated a number of classic case studies involving natural selection and clines, intramolecular coevolution and repeated sequence evolution. His star burned particularly brightly during the 1990's, but he consolidated this success with evolutionary and functional studies of ion channel genes and later applied what he had learned as a graduate student and then as a postdoc, to vector biology at the Oswaldo Cruz Institute (IOC). He initiated the molecular analysis of vector clocks and used clock gene sequences that evolve rapidly, to determine the evolutionary relationships among closely related vector populations, particularly in sandflies. Apart from his outstanding intellect (I never had a conversation about science with him when I didn't learn something - CPK/I have never met anyone brighter, more open-minded and willing to discuss when it comes to science - ACAMF), his enthusiasm for science and his easy-going outlook on life, provided his group at IOC with a mentor who was always approachable, helpful and fun to be around. His kindness, humour and generosity will always be remembered by his family, friends and colleagues and he is sadly missed by us all.

\section{REFERENCES}

Allada R, White NE, So WV, Hall JC, Rosbash M 1998. A mutant Drosophila homolog of mammalian clock disrupts circadian rhythms and transcription of period and timeless. Cell 93: 791-804.

Ampleford EJ, Davey KG 1989. Egg laying in the insect Rhodnius prolixus is timed in a circadian fashion. Journal of Insect Physiology 35: 183-188.

Ampleford EJ, Steel CGH 1982. Circadian control of ecdysis in Rhodnius prolixus (Hemiptera). J Comp Physiol 147: 281-286.

Anderson JM, Oliveira F, Kamhawi S, Mans BJ, Reynoso D, Seitz AE, Lawyer P, Garfield M, Pham M, Valenzuela JG 2006. Comparative salivary gland transcriptomics of sandfly vectors of visceral leishmaniasis. BMC Genomics 7: 52.

Azevedo RVDM, Dias DBS, Bretãs JAC, Mazzoni CJ, Souza NA, Albano RM, Wagner G, Dávila AMR, Peixoto AA 2012. The transcriptome of Lutzomyia longipalpis (Diptera: Psychodidae) male reproductive organs. PLOS ONE 7: e34495.

Bae K, Lee C, Hardin PE, Edery I 2000. dCLOCK is present in limiting amounts and likely mediates daily interactions between the dCLOCK-CYC transcription factor and the PER-TIM complex. J Neurosci 20: 1746-1753.
Bae K, Lee C, Sidote D, Chuang KY, Edery I 1998. Circadian regulation of a Drosophila homolog of the mammalian clock gene: PER and TIM function as positive regulators. Mol Cell Biol 18: 6142-151.

Baker CL, Loros JJ, Dunlap JC 2012. The circadian clock of Neurospora crassa. FEMS Microbiol Rev 36: 95-110.

Barrozo RB, Minoli SA, Lazzari CR 2004a. Circadian rhythm of behavioural responsiveness to carbon dioxide in the blood-sucking bug Triatoma infestans (Heteroptera: Reduviidae). $J$ Insect Physiol 50: 249-254.

Barrozo RB, Schilman PE, Minoli SA, Lazzari CR 2004b. Daily rhythms in disease-vector insects. Biol Rhythm Res 35: 79-92.

Bodin A, Barrozo RB, Couton L, Lazzari CR 2008. Temporal modulation and adaptive control of the behavioural response to odours in Rhodnius prolixus. J Insect Physiol 54: 1343-1348.

Bünning E 1936. Die endogene tagesperiodik als grundlage der photoperiodischen reaktion. Ber Dtsch Bot Ges 54: 590-608.

Busza A, Emery-Le M, Rosbash M, Emery P 2004. Roles of the two Drosophila CRYPTOCHROME structural domains in circadian photoreception. Science 304: 1503-1506.

Ceriani MF, Darlington TK, Staknis D, Más P, Petti AA, Weitz CJ, Kay SA 1999. Light-dependent sequestration of TIMELESS by CRYPTOCHROME. Science 285: 553-556.

Ceriani MF, Hogenesch JB, Yanovsky M, Panda S, Straume M, Kay SA 2002. Genome-wide expression analysis in Drosophila reveals genes controlling circadian behavior. J Neurosci 22: 9305-9319.

Chiba Y, Uki M, Kawasaki Y, Matsumoto A, Tomioka K 1993. Entrainability of circadian activity of the mosquito Culex pipiens pallens to 24-hr temperature cycles, with special reference to involvement of multiple oscillators. J Biol Rhythms 8: 211-220.

Chiba Y, Yamamoto Y, Shimizu C, Zaitsu M, Uki M, Yoshii M, Tomioka K 1990. Insemination-dependent modification of circadian activity of the mosquito, Culex pipiens pallens. Zool Sci 7: 895-906.

Claridge-Chang A, Wijnen H, Naef F, Boothroyd C, Rajewsky N, Young MW 2001. Circadian regulation of gene expression systems in the Drosophila head. Neuron 32: 657-671.

Clements AN 1999. The biology of mosquitoes, CABI, Wallingford, $740 \mathrm{pp}$.

Collins BH, Rosato E, Kyriacou CP 2004. Seasonal behavior in Drosophila melanogaster requires the photoreceptors, the circadian clock and phospholipase C. Proc Natl Acad Sci USA 101: 19451950.

Curtin KD, Huang ZJ, Rosbash M 1995. Temporally regulated nuclear entry of the Drosophila period protein contributes to the circadian clock. Neuron 14: 365-372.

Cyran SA, Buchsbaum AM, Reddy KL, Lin M-C, Glossop NRJ, Hardin PE, Young MW, Storti RV, Blau J 2003. vrille, Pdp1 and $d$ Clock form a second feedback loop in the Drosophila circadian clock. Cell 112: 329-341.

Darlington TK, Wager-Smith K, Ceriani MF, Staknis D, Gekakis N, Steeves TD, Weitz CJ, Takahashi JS, Kay SA 1998. Closing the circadian loop: CLOCK-induced transcription of its own inhibitors per and tim. Science 280: 1599-1603.

Das S, Dimopoulos G 2008. Molecular analysis of photic inhibition of blood-feeding in Anopheles gambiae. BMC Physiol 8: 23.

de Mairan J 1729. Observation botanique. In Histoire de l'Academie Royale des Sciences, ARS, Paris, p. 35-36.

Deane LM, Deane MP 1962. Visceral leishmaniasis in Brazil: geographical distribution and trnsmission. Rev Inst Med Trop Sao Paulo 4: 198-212. 
Dibner C, Schibler U, Albrecht U 2010. The mammalian circadian timing system: organization and coordination of central and peripheral clocks. Annu Rev Physiol 72: 517-549.

Dillon RJ, Ivens AC, Churcher C, Holroyd N, Quail MA, Rogers ME, Soares MB, Bonaldo MF, Casavant TL, Lehane MJ, Bates PA 2006. Analysis of ESTs from Lutzomyia longipalpis sand flies and their contribution toward understanding the insect-parasite relationship. Genomics 88: 831-840.

Dong G, Kim Y-I, Golden SS 2010. Simplicity and complexity in the cyanobacterial circadian clock mechanism. Curr Opin Genet Dev 20: 619-625.

Eckel-Mahan K, Sassone-Corsi P 2013. Metabolism and the circadian clock converge. Physi Rev 93: 107-135.

Edgar RS, Green EW, Zhao Y, van Ooijen G, Olmedo M, Qin X, Xu Y, Pan M, Valekunja UK, Feeney KA, Maywood ES, Hastings $\mathrm{MH}$, Baliga NS, Merrow M, Millar AJ, Johnson CH, Kyriacou CP, O’Neill JS, Reddy AB 2012. Peroxiredoxins are conserved markers of circadian rhythms. Nature 485: 459-464.

El-Din El-Assal S, Alonso-Blanco C, Peeters AJ, Raz V, Koornneef M 2001. A QTL for flowering time in Arabidopsis reveals a novel allele of CRY2. Nat Genet 29: 435-440.

Emery P, So WV, Kaneko M, Hall JC, Rosbash M 1998. CRY, a Drosophila clock and light-regulated cryptochrome, is a major contributor to circadian rhythm resetting and photosensitivity. Cell 95: 669-679.

Feliciangeli MD 2004. Natural breeding places of phlebotomine sandflies. Med Vet Entomol 18: 71-80.

Fuchikawa T, Sanada S, Nishio R, Matsumoto A, Matsuyama T, Yamagishi M, Tomioka K, Tanimura T, Miyatake T 2010. The clock gene cryptochrome of Bactrocera cucurbitae (Diptera: Tephritidae) in strains with different mating times. Heredity 104: 387-392.

Gallego M, Virshup DM 2007. Post-translational modifications regulate the ticking of the circadian clock. Nat Rev Mol Cell Biol 8: 139-148.

Gary RE, Foster WA 2006. Diel timing and frequency of sugar feeding in the mosquito Anopheles gambiae, depending on sex, gonotrophic state and resource availability. Med Vet Entomol 20: 308-316.

Gekakis N, Saez L, Delahaye-Brown A, Myers M, Sehgal A, Young MW, Weitz C 1995. Isolation of timeless by PER protein-interaction - defective interaction between timeless protein and longperiod mutant PER(L). Science 270: 811-815.

Gentile C, Meireles-Filho ACA, Britto C, Lima JBP, Valle D, Peixoto AA 2006. Cloning and daily expression of the timeless gene in Aedes aegypti (Diptera:Culicidae). Insect Biochem Molec Biol 36: $878-884$.

Gentile C, Rivas GBS, Meireles-Filho ACA, Lima JBP, Peixoto AA 2009. Circadian expression of clock genes in two mosquito disease vectors: cry2 is different. $J$ Biol Rhythms 24: 444-451.

Giebultowicz JM 2000. Molecular mechanism and cellular distribution of insect circadian clocks. Annu Rev Entomol 45: 769-793.

Giebultowicz JM, Stanewsky R, Hall JC, Hege DM 2000. Transplanted Drosophila excretory tubules maintain circadian clock cycling out of phase with the host. Curr Biol 10: 107-110.

Glossop NRJ, Houl JH, Zheng H, Ng FS, Dudek SM, Hardin PE 2003. VRILLE feeds back to control circadian transcription of clock in the Drosophila circadian oscillator. Neuron 37: 249-261.

Goto S, Denlinger D 2002. Short-day and long-day expression patterns of genes involved in the flesh fly clock mechanism: period, timeless, cycle and cryptochrome. J Insect Physiol 48: 803-816.
Hao H, Allen D, Hardin PE 1997. A circadian enhancer mediates PER-dependent mRNA cycling in Drosophila melanogaster. Mol Cell Biol 17: 3687-3693.

Hawking F 1975. Circadian and other rhythms of parasites. Adv Parasitol 13:123-182.

Helfrich-Förster C 2004. The circadian clock in the brain: a structural and functional comparison between mammals and insects. J Comp Physiol A 190: 601-613.

Helfrich-Förster C 2005. Neurobiology of the fruit fly's circadian clock. Genes Brain Behav 4: 65-76.

Helfrich-Förster C, Shafer OT, Wulbeck C, Grieshaber E, Rieger D, Taghert P 2007. Development and morphology of the clock-geneexpressing lateral neurons of Drosophila melanogaster. J Comp Neurol 500: 47-70.

Hunter-Ensor M, Ousley A, Sehgal A 1996. Regulation of the Drosophila protein timeless suggests a mechanism for resetting the circadian clock by light. Cell 84: 677-685.

Hurd H 2003. Manipulation of medically important insect vectors by their parasites. Annu Rev Entomol 48: 141-161.

Ivanchenko M, Stanewsky R, Giebultowicz JM 2001. Circadian photoreception in Drosophila: functions of cryptochrome in peripheral and central clocks. J Biol Rhythms 16: 205-215.

Jochim RC, Teixeira CR, Laughinghouse A, Mu J, Oliveira F, Gomes RB, Elnaiem D-E, Valenzuela JG 2008. The midgut transcriptome of Lutzomyia longipalpis: comparative analysis of cDNA libraries from sugar-fed, blood-fed, post-digested and Leishmania infantum chagasi-infected sand flies. BMC Genomics 9: 15.

Jones MDR 1981. The programming of circadian flight-activity in relation to mating and the gonotrophic cycle in the mosquito, Aedes aegypti. Physiol Entomol 6: 307-313.

Jones MDR 1982. Coupled oscillators controlling circadian flight activity in the mosquito, Culex pipiens quinquefasciatus. Physiol Entomol 7: 281-289.

Jones MDR, Gubbins SJ 1978. Changes in the circadian flight activity of the mosquito Anopheles gambiae in relation to insemination, feeding and oviposition. Physiol Entomol 3: 213-220.

Jones MDR, Gubbins SJ 1979. Modification of female circadian flightactivity by a male accessory gland pheromone in the mosquito, Culex pipiens quinquefasciatus. Physiol Entomol 4: 345-351.

Jones MDR, Cubbin CM, Marsh D 1972. Light-on effects and the question of bimodality in the circadian flight activity of the mosquito Anopheles gambiae. J Exp Biol 57: 347-357.

Keegan KP, Pradhan S, Wang J-P, Allada R 2007. Meta-analysis of Drosophila circadian microarray studies identifies a novel set of rhythmically expressed genes. PLoS Comput Biol 3: e208.

Klowden MJ, Zwiebel LJ 2004. Vector olfaction and behavior. In Biology of disease vectors, 2nd ed., Elsevier Academic Press, Burlington, p. 277-287.

Koh K, Zheng X, Sehgal A 2006. JETLAG resets the Drosophila circadian clock by promoting light-induced degradation of TIMELESS. Science 312: 1809-1812.

Konopka RJ, Benzer S 1971. Clock mutants of Drosophila melanogaster. Proc Natl Acad Sci USA 68: 2112-2116.

Kula-Eversole E, Nagoshi E, Shang Y, Rodriguez J, Allada R, Rosbash M 2010. Surprising gene expression patterns within and between PDF-containing circadian neurons in Drosophila. Proc Natl Acad Sci USA 107: 13497-13502.

Lazzari CR 1992. Circadian organization of locomotion activity in the haematophagous bug Triatoma infestans. J Insect Physiol 38: 895-903. 
Lazzari CR, Minoli SA, Barrozo RB 2004. Chemical ecology of insect vectors: the neglected temporal dimension. Trends Parasitol 20: 506-507.

Lee C, Bae K, Edery I 1998. The Drosophila CLOCK protein undergoes daily rhythms in abundance, phosphorylation and interactions with the PER-TIM complex. Neuron 21: 857-867.

Lee C, Bae K, Edery I 1999. PER and TIM inhibit the DNA binding activity of a Drosophila CLOCK-CYC/dBMAL1 heterodimer without disrupting formation of the heterodimer: a basis for circadian transcription. Mol Cell Biol 19: 5316-5325.

Lee Y, Montell C 2013. Drosophila TRPA1 functions in temperature control of circadian rhythm in pacemaker neurons. $J$ Neurosci 33: 6716-6725.

Lin Y, Han M, Shimada B, Wang L, Gibler TM, Amarakone A, Awad TA, Stormo GD, Van Gelder RN, Taghert PH 2002. Influence of the period-dependent circadian clock on diurnal, circadian and aperiodic gene expression in Drosophila melanogaster. Proc Natl Acad Sci USA 99: 9562-9567.

Lorenzo MG, Lazzari CR 1998. Activity pattern in relation to refuge exploitation and feeding in Triatoma infestans (Hemiptera: Reduviidae). Acta Trop 70: 163-170.

Lorenzo MG, Lazzari CR 1999. Temperature and relative humidity affect the selection of shelters by Triatoma infestans, vector of Chagas disease. Acta Trop 72: 241-249.

Majercak J, Chen W-F, Edery I 2004. Splicing of the period gene 3'terminal intron is regulated by light, circadian clock factors and phospholipase C. Mol Cell Biol 24: 3359-3372.

Martinek S, Inonog S, Manoukian AS, Young MW 2001. A role for the segment polarity gene shaggy/GSK-3 in the Drosophila circadian clock. Cell 105: 769-779.

Massouras A, Waszak SM, Albarca-Aguilera M, Hens K, Holcombe W, Ayroles JF, Dermitzakis ET, Stone EA, Jensen JD, Mackay TFC, Deplancke B 2012. Genomic variation and its impact on gene expression in Drosophila melanogaster. PLoS Genet 8: e1003055

McDonald M, Rosbash M 2001. Microarray analysis and organization of circadian gene expression in Drosophila. Cell 107: 567-578.

Meireles-Filho ACA, Amoretty PR, Souza NA, Kyriacou CP, Peixoto AA 2006a. Rhythmic expression of the cycle gene in a hematophagous insect vector. BMC Mol Biol 7: 38 .

Meireles-Filho ACA, da S Rivas GB, Gesto JSM, Machado RC, Britto C, de Souza NA, Peixoto AA 2006b. The biological clock of an hematophagous insect: locomotor activity rhythms, circadian expression and downregulation after a blood meal. FEBS Lett 580: 2-8.

Meireles-Filho ACA, Stark A 2009. Comparative genomics of gene regulation-conservation and divergence of cis-regulatory information. Curr Opin Genet Dev 19: 565-570.

Minoli SA, Lazzari CR 2003. Chronobiological basis of thermopreference in the haematophagous bug Triatoma infestans. J Insect Physiol 49: 927-932.

Moore-Ede MC, Sulzman FM, Fuller CA 1982. The clocks that time us, Harvard University Press, Cambridge.

Myers M, WagerSmith K, Rothenfluh-Hilfiker A, Young MW 1996. Light-induced degradation of TIMELESS and entrainment of the Drosophila circadian clock. Science 271: 1736-1740.

Nagel DH, Kay SA 2012. Complexity in the wiring and regulation of plant circadian networks. Curr Biol 22: R648-R657.

Naidoo N, Song W, Hunter-Ensor M, Sehgal A 1999. A role for the proteasome in the light response of the timeless clock protein. Science 285: 1737-1741.
Oliveira F, Jochim RC, Valenzuela JG, Kamhawi S 2009. Sand flies, Leishmania and transcriptome-borne solutions. Parasitol Int 58: $1-5$.

Ozkaya O, Rosato E 2012. The circadian clock of the fly: a neurogenetics journey through time. Adv Genet 77: 79-123.

Ozturk N, VanVickle-Chavez SJ, Akileswaran L, Van Gelder RN, Sancar A 2013. Ramshackle (Brwd3) promotes light-induced ubiquitylation of Drosophila Cryptochrome by DDB1-CUL4-ROC1 E3 ligase complex. Proc Natl Acad Sci USA 110: 4980-4985.

Peixoto AA, Gomes CA, Amoretty PR, Lins RM, Meireles-Filho AC, de Souza NA, Kyriacou CP 2001. New molecular markers for phlebotomine sand flies. Int J Parasitol 31: 635-639.

Peschel N, Veleri S, Stanewsky R 2006. Veela defines a molecular link between Cryptochrome and Timeless in the light-input pathway to Drosophila's circadian clock. Proc Natl Acad Sci USA 103: 17313-17318.

Pitaluga AN, Beteille V, Lobo AR, Ortigão-Farias JR, Dávila AMR, Souza AA, Ramalho-Ortigão JM, Traub-Cseko YM 2009. EST sequencing of blood-fed and Leishmania-infected midgut of Lutzomyia longipalpis, the principal visceral leishmaniasis vector in the Americas. Mol Genet Genomics 282: 307-317.

Pittendrigh CS 1950. The ecoclimatic divergence of Anopheles bellator and A. homunculus. Evolution 4: 43-63.

Pittendrigh CS 1954. on temperature independence in the clock system controlling emergence time in Drosophila. Proc Natl Acad Sci USA 40: 1018-1029.

Pittendrigh CS 1967. Circadian systems. I. The driving oscillation and its assay in Drosophila pseudoobscura. Proc Natl Acad Sci USA 58: $1762-1767$

Plautz JD, Kaneko M, Hall JC, Kay SA 1997. Independent photoreceptive circadian clocks throughout Drosophila. Science 278: $1632-1635$

Price JL, Blau J, Rothenfluh A, Abodeely M, Kloss B, Young MW 1998. Double-time is a novel Drosophila clock gene that regulates PERIOD protein accumulation. Cell 94: 83-95.

Ptitsyn AA, Reyes-Solis G, Saavedra-Rodriguez K, Betz J, Suchman EL, Carlson JO, Black WC 2011. Rhythms and synchronization patterns in gene expression in the Aedes aegypti mosquito. BMC Genomics 12: 153

Reisenman CE, Insausti TC, Lazzari CR 2002. Light-induced and circadian changes in the compound eye of the haematophagous bug Triatoma infestans (Hemiptera: Reduviidae). J Exp Biol 205: 201-210.

Reisenman CE, Lazzari CR, Giurfa M 1998. Circadian control of photonegative sensitivity in the haematophagous bug Triatoma infestans. J Comp Physiol 183: 533-541.

Rivas GBS, Souza NA, Peixoto AA 2008. Analysis of the activity patterns of two sympatric sandfly siblings of the Lutzomyia longipalpis species complex from Brazil. Med Vet Entomol 22: 288-290.

Rodriguez J, Tang C-HA, Khodor YL, Vodala S, Menet JS, Rosbash M 2013. Nascent-Seq analysis of Drosophila cycling gene expression. Proc Natl Acad Sci USA 110: 275-284.

Roubaud E 1918. Rhythmes physiologiques et vol spontané chez l'Anopheles maculipennis. CR Hebdomadaires des Séances de l'Academie des Science Paris 167: 967-969.

Rund SSC, Gentile JE, Duffield GE 2013. Extensive circadian and light regulation of the transcriptome in the malaria mosquito Anopheles gambiae. BMC Genomics 14: 218.

Rund SSC, Hou TY, Ward SM, Collins FH, Duffield GE 2011. Genome-wide profiling of diel and circadian gene expression in the 
malaria vector Anopheles gambiae. Proc Natl Acad Sci USA 108: 421-430.

Rund SSC, Lee SJ, Bush BR, Duffield GE 2012. Strain and sex-specific differences in daily flight activity and the circadian clock of Anopheles gambiae mosquitoes. J Insect Physiol 58: 1609-1619.

Rush BL, Murad A, Emery P, Giebultowicz JM 2006. Ectopic CRYPTOCHROME renders TIM light sensitive in the Drosophila ovary. J Biol Rhythms 21: 272-278.

Rutila JE, Suri V, Le M, So WV, Rosbash M, Hall JC 1998. CYCLE is a second bHLH-PAS clock protein essential for circadian rhythmicity and transcription of Drosophila period and timeless. Cell 93: 805-814.

Sakai T, Tamura T, Kitamoto T, Kidokoro Y 2004. A clock gene, period, plays a key role in long-term memory formation in Drosophila. Proc Natl Acad Sci USA 101: 16058-16063.

Sandrelli F, Costa R, Kyriacou CP, Rosato E 2008. Comparative analysis of circadian clock genes in insects. Insect Mol Biol 17: 447-463.

Sarov-Blat L, So WV, Liu L, Rosbash M 2000. The Drosophila takeout gene is a novel molecular link between circadian rhythms and feeding behavior. Cell 101: 647-656.

Sathyanarayanan S, Zheng X, Xiao R, Sehgal A 2004. Posttranslational regulation of Drosophila PERIOD protein by protein phosphatase 2A. Cell 116: 603-615.

Saunders DS 2002. Insect Clocks, 3rd ed., Elsevier, Amsterdam, 560 pp.

Schaub GA 2006. Parasitogenic alterations of vector behaviour. Int $J$ Med Microbiol 296 (Suppl.) 40: 37-40.

Sehadova H, Glaser FT, Gentile C, Simoni A, Giesecke A, Albert JT, Stanewsky R 2009. Temperature entrainment of Drosophila's circadian clock involves the gene nocte and signaling from peripheral sensory tissues to the brain. Neuron 64: 251-266.

Severson DW, Behura SK 2012. Mosquito genomics: progress and challenges. Annu Rev Entomol 57: 143-166.

So WV, Rosbash M 1997. Post-transcriptional regulation contributes to Drosophila clock gene mRNA cycling. EMBO J 16: 7146-7155.

So WV, Sarov-Blat L, Kotarski CK, McDonald M, Allada R, Rosbash M 2000. Takeout, a novel Drosophila gene under circadian clock transcriptional regulation. Mol Cell Biol 20: 6935-6944.

Stanewsky R, Kaneko M, Emery P, Beretta B, Wager-Smith K, Kay SA, Rosbash M, Hall JC 1998. The cryb mutation identifies cryptochrome as a circadian photoreceptor in Drosophila. Cell 95: 681-692.

Steel CGH, Vafopoulou X 2006. Circadian orchestration of developmental hormones in the insect, Rhodnius prolixus. Comp Biochem Physiol A Mol Integr Physiol 144: 351-364.

Tanoue S, Krishnan P, Krishnan B, Dryer SE, Hardin PE 2004. Circadian clocks in antennal neurons are necessary and sufficient for olfaction rhythms in Drosophila. Curr Biol 14: 638-649.

Tauber E, Roe H, Costa R, Hennessy JM, Kyriacou CP 2003. Temporal mating isolation driven by a behavioral gene in Drosophila. Curr Biol 13: 140-145.

Taylor B, Jones MD 1969. The circadian rhythm of flight activity in the mosquito Aedes aegypti (L.). The phase-setting effects of light-on and light-off. J Exp Biol 51: 59-70.

Tomioka K, Uryu O, Kamae Y, Umezaki Y, Yoshii T 2012. Peripheral circadian rhythms and their regulatory mechanism in insects and some other arthropods: a review. J Comp Physiol B Biochem Syst Environ Physiol 182: 729-740.

Ueda HR, Matsumoto A, Kawamura M, Iino M, Tanimura T, Hashimoto S 2002. Genome-wide transcriptional orchestration of circadian rhythms in Drosophila. J Biol Chem 277: 14048-14052.

Vafopoulou X, Steel C 2001. Induction of rhythmicity in prothoracicotropic hormone and ecdysteroids in Rhodnius prolixus: roles of photic and neuroendocrine zeitgebers. J Insect Physiol 47: 935-941.

Vafopoulou X, Steel CG 1996. Circadian regulation of a daily rhythm of release of prothoracicotropic hormone from the brain retrocerebral complex of Rhodnius prolixus (Hemiptera) during larvaladult development. Gen Com Endocrinol 102: 123-129.

Vafopoulou X, Steel CGH 2006. Ecdysteroid hormone nuclear receptor (EcR) exhibits circadian cycling in certain tissues, but not others, during development in Rhodnius prolixus (Hemiptera). Cell Tissue Res 323: 443-455.

Vafopoulou X, Terry KL, Steel CGH 2010. The circadian timing system in the brain of the fifth larval instar of Rhodnius prolixus (Hemiptera). J Comp Neurol 518: 1264-1282.

Vanin S, Bhutani S, Montelli S, Menegazzi P, Green EW, Pegoraro M, Sandrelli F, Costa R, Kyriacou CP 2012. Unexpected features of Drosophila circadian behavioural rhythms under natural conditions. Nature 484: 371-375.

Warren JT, Sakurai S, Rountree DB, Gilbert LI, Lee SS, Nakanishi K 1988. Regulation of the ecdysteroid titer of Manduca sexta: reappraisal of the role of the prothoracic glands. Proc Natl Acad Sci USA 85: 958-962.

Wheeler DA, Hamblen-Coyle MJ, Dushay MS, Hall JC 1993. Behavior in light-dark cycles of Drosophila mutants that are arrhythmic, blind or both. J Biol Rhythms 8: 67-94.

Wolfgang W, Simoni A, Gentile C, Stanewsky R 2013. The Pyrexia transient receptor potential channel mediates circadian clock synchronization to low temperature cycles in Drosophila melanogaster. Proc Biol Sci 280: 20130959.

Xu K, Diangelo JR, Hughes ME, Hogenesch JB, Sehgal A 2011. The circadian clock interacts with metabolic physiology to influence reproductive fitness. Cell Metab 13: 639-654.

Yang Y-Y, Liu Y, Teng H-J, Sauman I, Sehnal F, Lee H-J 2010. Circadian control of permethrin-resistance in the mosquito Aedes aegypti. J Insect Physiol 56: 1219-1223.

Yu W, Zheng H, Houl JH, Dauwalder B, Hardin PE 2006. PER-dependent rhythms in CLK phosphorylation and E-box binding regulate circadian transcription. Genes Dev 20: 723-733.

Yuan Q, Metterville D, Briscoe AD, Reppert SM 2007. Insect cryptochromes: gene duplication and loss define diverse ways to construct insect circadian clocks. Mol Biol Evol 24: 948-955.

Zheng H, Ng F, Liu Y, Hardin PE 2008. Spatial and circadian regulation of cry in Drosophila. J Biol Rhythms 23: 283-295.

Zeng H, Qian Z, Myers MP, Rosbash M 1996. A light-entrainment mechanism for the Drosophila circadian clock. Nature 380: 129-135.

Zhu H, Sauman I, Yuan Q, Casselman A, Emery-Le M, Emery P, Reppert SM 2008. Cryptochromes define a novel circadian clock mechanism in monarch butterflies that may underlie sun compass navigation. Plos Biol 6: e4.

Zhu H, Yuan Q, Briscoe AD, Froy O, Casselman AL, Reppert SM 2005. The two CRYs of the butterfly. Curr Biol 15: R953-R954. 